minimus (normal $>2.8 \mathrm{mV}$ ) and $0.66 \mathrm{mV}$ in the extensor digitorum brevis (normal $>2.5 \mathrm{mV}$ ) (figure). A follow up examination one year later again showed normal motor and sensory nerve conduction. Repetitive stimulation of the ulnar nerve at $3 \mathrm{~Hz}$ recording from the abductor digiti minimus showed a $17 \%$ decremental response and post-tetanic potentiation of $30 \%$. Compound muscle action potential amplitude of the abductor digiti minimus was $7 \cdot 5 \mathrm{mV}$.

Antibodies to $\mathrm{N}$-type calcium channels were assayed in the Institute of Molecular Medicine laboratories, Oxford, using ${ }^{125} \mathrm{I}-\mathrm{w}-$ conotoxin-labelled human neuroblastoma (SKN-SH) cells. ${ }^{5}$ Titres are expressed as $\mathrm{pM}$, values $>30 \mathrm{pM}$ being considered positive. ${ }^{5}$ The Figure shows serum antiVGCC antibody titres at four time points after the diagnosis of SCD. The titre was also measured in a CSF sample taken about six months after presentation. This titre was $42 \mathrm{pM}$; correcting for the IgG concentration of $0.02 \mathrm{mg} / \mathrm{ml}$ gave a normalised titre of $2100 \mathrm{pM} / \mathrm{g} \mathrm{IgG.}{ }^{2}$ A progressive decline in the serum anti-VGCC antibody titre was noted, concurrent with the increase in amplitude of the abductor digiti minimus compound muscle action potential amplitude, over the next 18 months (figure).

Two years after initiation of immunotherapy, the patient showed improvement of gait and strength, although diplopia and cerebellar dysfunction persisted.

This patient shows the clinical features of SCD and the clinical and electromyographic features of LEMS. Prior reports have shown a positive effect of plasma exchange and immunosuppressive treatment in patients with $\mathrm{LEMS}^{57}$ including a fall in the titre of anti-VGCC antibodies with immunosuppressive treatment associated with improved electrophysiological measures of disease activity. ${ }^{5}$ With immunosuppressive treatment our patient showed improvement in neuromuscular, but not cerebellar, symptoms and this improvement was associated with decreasing serum titres of anti-VGCC antibodies (figure).

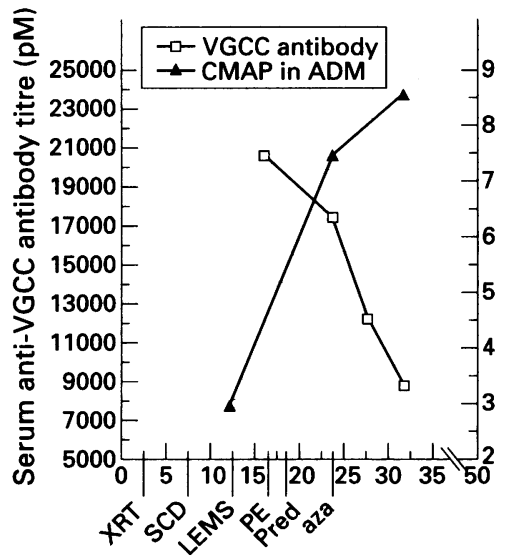

Time from onset of lymphoma (months)

Serum anti-VGCC antibody titre and compound muscle action potential amplitude in the abductor digiti minimus (ADM) with regard to the onset of SCD and LEMS. XRT = radiotherapy; $C M A P=$ compound muscle action potential; pred $=$ predisore; $a z a=$ azathioprine; $P E=$ plasma exchange.
The finding of raised antibody titres to VGCC in patients with LEMS, and in patients with SCD associated with LEMS, raises the possibility that the two disorders are due to autoantibodies of similar specificities. ${ }^{128}$ The presence of anti-VGCC antibodies in both serum and CSF in our patient is consistent with this hypothesis. The development of SCD in only a subset of patients with LEMS suggests that if both are due to the same autoantibody, the ability of the antibody to enter the CNS may differ between patients. This could be due to higher serum titres of anti-VGCC antibodies in patients with SCD and LEMS, or to intrathecal antibody production as occurs in multiple sclerosis. ${ }^{9}$ The second possibility is supported by the presence of oligoclonal immunoglobulin bands in the CSF.

The lack of effect of immunosuppresive treatment on the symptoms due to SCD, by contrast with those due to LEMS, may be because of irreversible damage to Purkinje cells. Alternatively, the immunosuppressive regimens used to date may be ineffective in attenuating the immune response within the CNS. This could be because antibody forming cells making pathogenic antibody have taken up residence inside the bloodbrain barrier, where they may be less accessible to immunosuppressive treatment. It is also possible that cerebellar neurons are affected differently by anti-VGCC antibodies than presynaptic terminals at the motor endplate. Neurons express a number of subtypes of VGCCs, ${ }^{10}$ some of which are expressed preferentially by cerebellar Purkinje cells. ${ }^{11}$

The pattern of combined central and peripheral nervous system involvement in this patient, together with increased antibody titres to VGCCs and a differential response of neuromuscular symptoms to immunosuppression, suggests that VGCC antibodies may play a differential role in the pathogenesis of the peripheral and central deficits in LEMS and SCD.

J M GOLDSTEIN S G WAXMAN

Department of Neurology, Yale University School of Medicine, New Haven, CT, USA B LANG

I JOHNSTON J NEWSOM-DAVIS Neurosciences Group, Institute of Molecular Medicine, University of Oxford, Fohn Radcliffe Hospital,
Oxford, UK

Correspondence to: Dr JM Goldstein, Department of Neurology, Yale University School of Medicine, 333 Cedar Street, New Haven, CT 06510-3289, USA

1 Blumenfeld AM, Recht LD, Chad DA DeGirolami U, Griffin T, Jaeckle KA DeGirolami of Griffin T, Jaeckle KA syndrome and subacute cerebellar degenerasyndrome and subacute cerebellar degeneration: differential effects
Neurology 1991;41:1682-5.

2 Clouston PD, Saper CB, Arbizu T, et al. Paraneoplastic cerebellar degeneration. III. Cerebellar degeneration, cancer, and the Lambert-Eaton myasthenic syndrome. Neurology 1992;42:1944-50.

3 O'Neill JH, Murray NMF, Newsom-Davis J. The Lambert-Eaton myasthenic syndrome a review of 50 cases. Brain 1988;111 577-96.

4 Lang B, Newsom-Davis J, Prior C, Wray D. Antibodies to motor nerve terminals: an electrophysiological study of a human myasthenic syndrome transferred to mouse. my Physiol 1983;344:335-45.

5 Leys K, Lang B, Johnston I, Newsom-Davis J. Calcium channel autoantibodies in the Cambert-Eaton myasthenic syndrome. Ann Leurol 1991;29:307-14.

6 Lennon VA, Lambert EH. Autoantibodies bind solubilized calcium channel-w-conotoxin complexes from small cell lung carcinoma: a diagnostic aid for Lambert-Eaton myasthenic syndrome. Mayo Clin Proc 1989; 64:1498-1504.

7 Newsom-Davis J, Murray NMF. Plasma exchange and immunosuppressive drug treatment in the Lambert-Eaton myas
syndrome. Neurology 1984;34:480-5.

8 Oguro-Okano M, Griesmann GE, Wieben ED, Slaymaker SJ, Snutch TP, Lennon VA. Molecular diversity of neuronal-type calcium channels identified in small cell lung carcinoma. Mayo Clin Proc 1992;67:1150-9.

9 Cutler RWP, Watters GV, Hammerstad JP. The origin and turnover rates of cerebrospinal fluid albumin and gamma globulin in man. F Neurol Sci 1970;10:259.

10 Miller RJ. Multiple calcium channels and neuronal function. Science 1987;235:46-52.

11 Llinás RR. The intrinsic electrophysiological properties of mammalian neurons: insights into central nervous system functions. Science 1988;242:1654-64.

Benign thalamic cyst presenting with contralateral postural tremor

Postural or action tremors have several aetiologies, but intracranial masses are rarely implicated as a cause. We present a young girl with a cystic mass of the right thalamus whose sole presenting complaint was a unilateral postural tremor affecting the left upper and lower limbs.

An 11 year old girl presented with involuntary movements of the left upper limb for seven months and the left lower limb for five months. She had no history of weakness of these limbs, gait disturbance, or symptoms of raised intracranial pressure. On examination she had no papilloedema or weakness of any of her limbs. There was a coarse postural tremor $(6-8 \mathrm{~Hz})$ of the outstretched left upper limb, which was more prominent in the distal muscle groups. On attempting to stand or walk, the left lower limb also manifested the tremor; it was most evident in the ankle and foot. The tremor was accentuated slightly with movement of the limbs and seemed to have a greater amplitude while nearing the target in the finger to nose test. The tremor was abolished when the limbs were at rest and when the patient was asleep. She had no nystagmus or gait ataxia and had a normal muscle tone and a normal speech. There was no dystonic posturing or sensory abnormalities.

CT of the head showed a large, well defined, rounded, non-enhancing, hypodense mass occupying the right thalamus with the same density as CSF. The lateral and third ventricles were normal sized. MRI suggested the presence of fluid in the mass with an intensity similar to that of CSF (figure (A), and (B)). A CT guided stereotactic aspiration of the cyst yielded $45 \mathrm{ml}$ of clear, colourless fluid. As there was no enhancing wall a target was not chosen for biopsy. Post operative CT showed pronounced reduction in the size of the cyst with no blood within or outside it. Analysis of the fluid showed protein $47 \mathrm{mg} / \mathrm{dl}$, sugar $33 \mathrm{mg} / \mathrm{dl}$, and 2 lymphocytes per $\mathrm{mm}^{3}$. The fluid was centrifuged and the sediment examined for cysticercus scolices, hydatid sand, and malignant cells, all of which were negative. A diagnosis of a benign epithelial (ependymal) cyst was made. ${ }^{1}$ There was a dramatic improvement in her symptoms in the immediate postoperative period with virtual cessation of the tremors. 


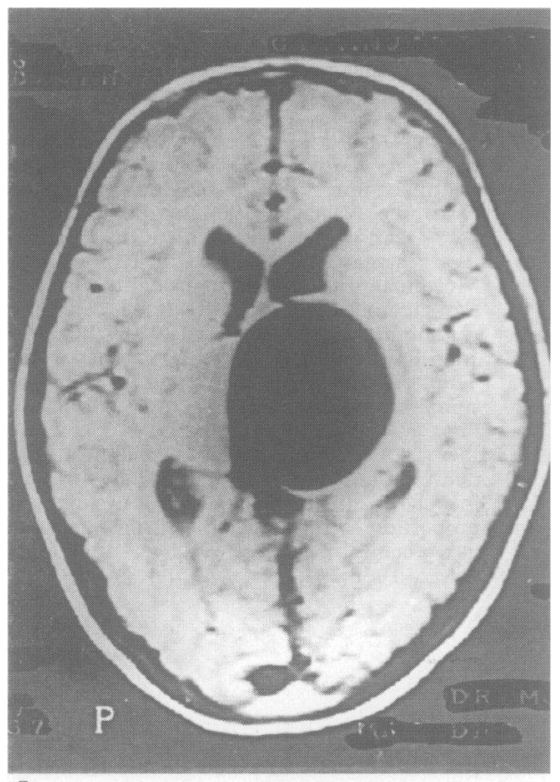

$\mathbf{A}$

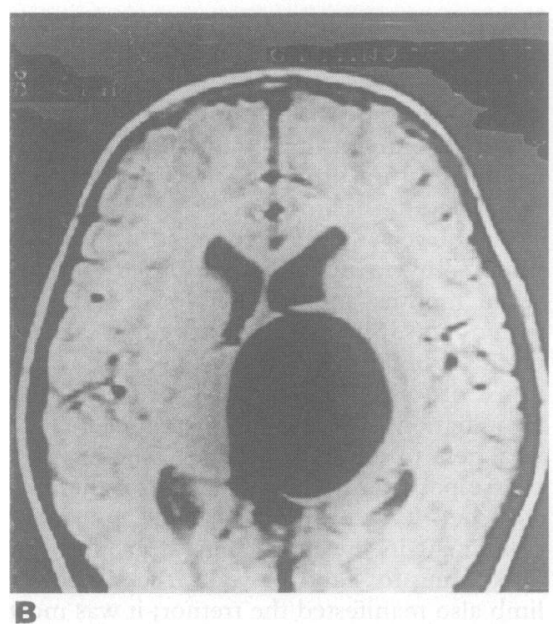

$T 1$ weighted axial $(A)$ and sagittal (B) MRI of the patient showing the hypointense cystic lesion in the right thalamus and basal ganglia. Compression of the upper midbrain by the mass is evident in the sagittal scan.

At follow up three months after surgery, the patient was asymptomatic and had no tremors of the left upper or lower limbs. A small residual cyst was seen on CT but it was not producing any mass effect or ventricular dilatation. No further treatment was offered and she was advised clinical and CT monitoring.

Intracranial tumours are on occasions known to produce a parkinsonian syndrome with rest tremors, bradykinesia, and rigidity. ${ }^{23}$ Another type of tremor that has been described in association with intracranial masses is "rubral tremor". Involvement of the red nucleus and the decussating fibres of the superior cerebellar peduncle by lesions in the midbrain region has been implicated in the production of "rubral tremor". ${ }^{4}$ In both these instances, the tremor is present at rest, although rubral tremors may be aggravated by maintenance of a posture or goal directed movement.

Postural tremors can theoretically occur in isolated cerebellar hemispheric lesions including mass lesions, but this manifestation is rarely seen in clinical practice. The cerebellar connections with the brainstem, especially the superior cerebellar peduncle, should be spared for a postural type of tremor to occur. An intention tremor results if these connections are involved. ${ }^{4}$ The postural tremor caused by cerebellar lesions is a slow tremor of 4 to $5 \mathrm{~Hz}$ and is thought to result from hypotonia of the affected limbs. ${ }^{4}$

The postural tremor in our case was unexpected considering the location of the mass in the thalamus with compression of the adjacent basal ganglia. The structures likely to be affected by the cyst include the basal ganglia and their connections, dentatorubrothalamic fibres, and the thalamic nuclei. Lesions of these structures are not known to produce isolated postural tremors. The mass in our patient could be expected to produce a rest tremor. Even an intention tremor is conceivable considering the possible compression of the superior cerebellar peduncle by the cyst. As mentioned, only discrete lesions of the cerebellar hemisphere have been considered to cause postural tremors and our patient had neither hypotonia nor involvement of the cerebellar hemispheres.

The tremor in our case was contralateral to the side of the tumour and responded to aspiration of the cyst contents, suggesting that the pressure exerted by the cyst on adjacent neural structures was in some way responsible for the production of the tremor. To the best of our knowledge there has been no previous report of unilateral postural tremor caused by a thalamic or basal ganglia lesion.

VEDANTAM RAJSHEKHAR Department of Neurological Sciences,

Christian Medical College and Hospital,
Vellore 632004, India

1 Friede RL, Yasargil MG. Supratentorial intracerebral epithelial (ependymal) cysts: review, case reports and fine structure. f Neurol Neurosurg Psychiatry 1977;40: 127-37.

2 de Yebenes GJ, Gervas JJ, Iglesias J, et al. Biochemical findings in a case of parkinsonism secondary to brain tumor. Ann Neurol 1981;11:313-6.

3 Liversedge LA. Involuntary movements. In: Vinken PJ, Bruyn GW, eds. Handbook of clinical neurology, Vol 1. Amsterdam: North Holland Publishing Co, 1969:277-92.

4 Lang AE. Movement disorder symptomatology. In: Bradley WG, Daroff RB, Fenichel GM, Marsden CD, eds. Neurology in clinical practice, Vol 1. Boston: Butterworths; practice, Vol 1. Boston:

\section{MRI of neuromyelitis optica: evidence for a distinct entity}

Neuromyelitis optica was originally described as a distinct demyelinating disorder characterised by visual disturbances and spinal cord signs occurring closely in time. ${ }^{1}$ Iater studies on patients presenting with this syndrome often reported the subsequent evolution of additional neurological deficits, ${ }^{2}$ found pathological evidence for a more widespread demyelination, or disclosed other specific aetiologies such as acute disseminated encephalomyelitis, Behcet's disease, or systemic lupus erythematosus. ${ }^{3}$ From these findings it was concluded by many that neuromyelitis optica was inseparable from multiple sclerosis and its existence as a specific entity was questioned. ${ }^{34} \mathrm{MRI}$ promises to alter this atti- tude, as we show by the presentation of two cases.

Starting in 1981 , case 1 , a 28 year old woman, had repeatedly experienced paraesthesias of the extremities and episodes of blurred vision. In 1988, during a further bout of visual deterioration MRI of the brain showed swelling and contrast enhancement of the optic chiasm and of the prechiasmatic portion of the optic nerves. An exploratory frontal craniotomy six months later showed atrophic optic nerves that were encased by abnormally thick arachnoidea but no tumour.

One year later the patient was first seen at our department for rapid onset of left leg weakness and bilaterally ascending hypaesthesia. At that time visual acuity was reduced to finger counting. MRI showed swelling of the upper thoracic cord with irregular intramedullary enhancement after giving gadolinium-DTPA. The brain seemed normal, including the chiasm. Lumbar puncture and a spinal angiogram were uninformative. Laboratory tests were negative for involvement of any other organ, systemic infection, or collagen vascular disease. No specific antibodies except for an intermittently raised titre against Toxoplasma gondii was found.

Over the next four years the patient continued to have bouts of myelitis at times involving almost the entire spinal cord, as shown by MRI. Cell counts in the CSF ranged from 0 to $10 / \mathrm{mm}^{3}$ and consisted mainly of lymphocytes and activated monocytes. Sometimes fat containing macrophages indicating tissue necrosis were also seen. The protein content of CSF was mostly increased and ranged from 71 to 153 $\mathrm{mg} / 100 \mathrm{ml}$, but there was no evidence of intrathecal immunoglobulin production. Repeated search for oligoclonal bands was negative.

Various therapeutic regimens including high dose steroids, immunoglobulins, immune adsorption, and antibiotic treatments failed to stabilise the patient's clinical condition. When she was last seen in mid19.92 she was bedridden because of a spastic tetraparesis with a bilateral sensory level at $\mathrm{C} 2$ and had unchanged severe visual impairment. MRI of the brain continued to be normal whereas the spinal cord at the cervical and upper thoracic region was very atrophic. The patient refused the administration of contrast material at that time.

For five months case 2, a 22 year old woman, had experienced continuous deterioration of visual acuity associated with increasing bilateral leg weakness and urinary incontinence before she was seen at our department. Visual field testing showed concentric narrowing on the right and a temporal hemianopia on the left with sparing of the fovea. Her right arm distal to the elbow was mildly hypaesthetic and sensation to pinprick and pain was reduced distally from $\mathrm{T} 7$ bilaterally. The plantar response was upgoing on both sides. A spinal tap gave a colourless CSF with 4 lymphocytes $/ \mathrm{mm}^{3}$ and a protein concentration of $50 \mathrm{mg} / 100 \mathrm{ml}$. There was no evidence of oligoclonal bands. MRI showed right sided enlargement of the chiasm and of the right optic nerve with uptake of contrast material. The remainder of the brain was normal. There was also minimal swelling of the cervical medulla and patchy, intramedullary enhancement from $\mathrm{Cl}$ to 
critically significant groups $\times$ measures interaction.

The effect that Rosser and Hodges claim may well be present in their data and, if so, would help to contribute to the understanding of the relation between allegedly cortical and subcortical dementias. Unfortunately the analyses actually reported do not properly permit the suggested conclusion to be drawn and it would therefore be useful to know if the relevant interaction really is significant.

$$
\begin{array}{r}
\text { E MILLER } \\
\text { Department of Psychology } \\
\text { (Clinical Section), } \\
\text { University of Leicester, } \\
\text { Leicester LE1 7RH, UK }
\end{array}
$$

1 Rosser A, Hodges JR. Initial letter and semantic category fluency in Alzheimer's sive supranuclear palsy. $\mathcal{F}$ Neurol Neurosurg sive supranuclear palsy. F Neurol
Psychiatry 1994;57:1389-1394.

2 Winer JB. Statistical principles in experimental design. 3rd ed. New York: McGraw mental design.
Hill, 1991.

Hodges replies:

We are very grateful for Miller's comments on our paper. We have now performed a split plot analysis of variance with both within subject and between subject effects, which confirmed the presence of a significant interaction $(F($ df 51,3$)=19 \cdot 6, P<$ $0.001)$. This highly significant group conditions interaction confirms the differential effect of Alzheimer's disease $v$ Huntington's disease and progressive supranuclear palsy on category and letter based fluency tests, which we hope will convince Professor Miller.

J R HODGES

Department of Neurology, Addenbrooke's Hospital, Hills Road, Cambridge CB2 2QQ, UK

Jarisch-Herxheimer reaction in a patient with neurosyphilis: nonconvulsive status epilepticus?

In their lesson of the month Zifko et al ${ }^{1}$ described a patient with neurosyphilis who developed fluctuating consciousness, disorientation, restlessness, and fever 10 hours after starting intravenous penicillin $\mathrm{G}$. $\mathrm{He}$ had tachycardia and hyperhydrosis. An EEG showed generalised 3/s rhythmic activity (periodic lateralised epileptiform discharges). These findings were attributed to a Jarisch-Herxheimer reaction.

We suggest that non-convulsive status epilepticus is an alternative explanation Altered consciousness and prolonged confusion are the central clinical findings of nonconvulsive status, which may occur without preceding or accompanying generalised tonic clonic seizures, may resolve spontaneously, and may be associated with the autonomic symptoms of fever and tachycardia. ${ }^{2}$ Periodic lateralised epileptiform discharges are one of the EEG correlates of non-convulsive status. ${ }^{4}$ As stated by the authors, patients with neurosyphilis are susceptible to epileptic seizures, both due to the condition itself ${ }^{5}$ and due to a JarischHerxheimer reaction. In addition, penicillin itself may precipitate seizures. ${ }^{6}$ In their patient, non-convulsive status may have been evoked by one of these mechanisms. It would be of value to know whether patients such as this respond to antiepileptic medication.

N HEYE JW DUNNE Royal Perth Hospica GPO Box X2213, Perth, Western Australia , Australia

1 Zifko U, Lindner K, Wimberger D, Volc B, Grisold W. Jarisch-Herxheimer reaction in a patient with neurosyphilis. $f$ Neurol Neurosurg Psychiatry 1994;57:865-7.

2 Dunne JW, Summers QA, Stewart-Wynne EG. Non-convulsive status epilepticus. A prospective study in an adult general hospi-

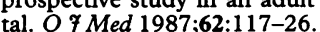

3 Cascino GD. Non convulsive status epilepticus in adults and children. Epilepsia 1993;34 (suppl 1):S21-28.

4 Grand'Maison F, Reiher J, Leduc CP. Retrospective inventory of EEG abnormalities in partial status epilepticus. Electroencephalogr Clin Neurophysiol 1991;79: 264-70.

5 Radhakrishon K, Ashok PP, Sridharan R, ElMangoush $M$. Periodic pattern in meningovascular syphilis. $f$ Neurol Neurosurg Psychiatry 1984;47:1360-1.

6 Davies DM. Textbook of adverse drug reactions. Chapter 18. Neurological disorders. 3rd ed. Oxford: Oxford University Press, 1985:495.

Zifko et al reply:

We appreciated the interesting response of Dune and Heye to our article. Although we cannot completely exclude the possibility of non-convulsive status epilepticus in our patient, we have several arguments that make their theory unlikely.

Firstly, autonomic symptoms of fever and tachycardia in our patient occurred before onset of confusion and altered consciousness, and were not present during the period of disorientation and psychomotor restlessness, as mentioned in the text. Secondly, although periodic lateralised epileptiform discharges may be a sign of non-convulsive status, this abnormality is non-specific. Thirdly, during CT, psychomotor restlessness was treated with 70 $\mathrm{mg}$ intravenous diazepam, which did not affect the confusion. Non-convulsive status of complex partial type usually responds well to diazepam.

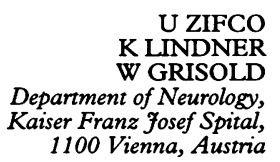

\section{Risperidone in Parkinson's disease}

A recent, excellent review of the management of Parkinson's disease called attention to the atypical neuroleptic drug clozapine (an antagonist of dopamine $\mathrm{D}_{4}$ and serotonin $5 \mathrm{HT}_{2}$ receptors) for amelioration of psychotic symptoms derived from dopaminergic treatments, when temporary withdrawal of antiparkinsonian drugs fails. Small doses of the atypical neuroleptic drug risperidone $(0.25-1.25 \mathrm{mg} /$ day) can also be used for ameliorating hallucinations induced by levodopa without worsening motor symptoms in Parkinson's disease. Risperidone has a strong affinity for $5 \mathrm{HT}$ receptors and only moderate affinity for $\mathrm{D}_{2}$ receptors. For parkinsonian patients in whom the $1-2 \%$ risk of agranulocytosis with clozapine is unacceptable, risperidone is another option

ALMIR R TAVARES, JR UFMG School of Medic Belo Horizonte, Brazil

1 Marsden CD. Parkinson's disease. 7 Neurol Neurosurg Psychiatry 1994;57:672-81.

2 Meco G, Alessandria A, Bonifati V, Giustini P. Risperidone for hallucinations in levodopatreated Parkinson's disease patients. Lancet 1994;343:1370-1.

\section{CORRECTION}

Rajshekhar V. Benign thalamic cyst presenting with contralateral postural tremor. I Neurol Neurosurg Psychiatry 1994;57:1139-40.

During production, the figure was incorrectly printed. The correct version is given here.
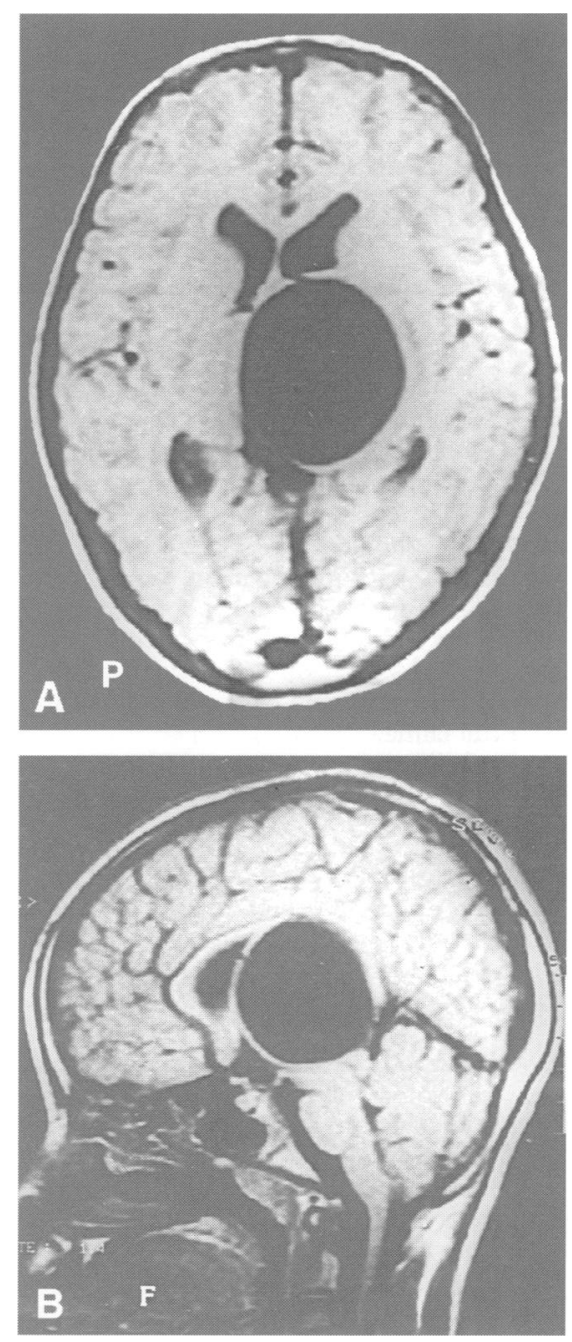

$T 1$ weighted axial $(A)$ and sagittal (B) MRI of the patient showing the hypointense cystic lesion in the right thalamus and basal ganglia. Compression of the upper midbrain by the mass is evident in the sagittal scan. 